\title{
Conceptualizing and treating social anxiety in Autism Spectrum Disorder: a focus group study with multidisciplinary professionals
}

Article

Accepted Version

Spain, D., Rumball, F., O'Neill, L., Sin, J., Prunty, J. and Happé, F. (2017) Conceptualizing and treating social anxiety in Autism Spectrum Disorder: a focus group study with multidisciplinary professionals. Journal of Applied Research in Intellectual Disabilities, 30 (S1). pp. 10-21. ISSN 1360-2322 doi: https://doi.org/10.1111/jar.12320 Available at https://centaur.reading.ac.uk/71141/

It is advisable to refer to the publisher's version if you intend to cite from the work. See Guidance on citing.

To link to this article DOI: http://dx.doi.org/10.1111/jar.12320

Publisher: Wiley

All outputs in CentAUR are protected by Intellectual Property Rights law, including copyright law. Copyright and IPR is retained by the creators or other copyright holders. Terms and conditions for use of this material are defined in the End User Agreement. 


\section{www.reading.ac.uk/centaur}

\section{CentAUR}

Central Archive at the University of Reading

Reading's research outputs online 


\section{Conceptualising and treating social anxiety in autism spectrum disorder:}

\section{A focus group study with multidisciplinary professionals}

\section{Authors' details}

Debbie Spain $^{\mathrm{ab}}$, Freya Rumball ${ }^{\mathrm{a}}$, Lucy O’Neill ${ }^{\mathrm{a}}$, Jacqueline $\mathrm{Sin}^{\mathrm{ac}}$, Jonathan Prunty ${ }^{\mathrm{a}}$ and Francesca Happé ${ }^{\mathrm{a}}$

a. Institute of Psychiatry, Psychology \& Neuroscience, King's College London

b. South London \& Maudsley NHS Foundation Trust

c. St George's, University of London

\section{Correspondence details}

Debbie Spain

Address: MRC Social, Genetic and Developmental Psychiatry Centre, Institute of Psychiatry, Psychology \& Neuroscience, King's College London, de Crespigny Park, PO Box 80, London, SE5 8AF, UK

Telephone: 00442078485388

Fax: 00442078480650

Email: debbie.spain@kcl.ac.uk

\section{Acknowledgements}

We would like to thank the participants who attended the focus groups. DS is funded by a National Institute for Health Research (NIHR) Clinical Doctoral Research Fellowship (CDRF-2012-03-059). This paper presents independent research funded by the NIHR. The views expressed are those of the authors and not necessarily those of the NHS, the NIHR, or the Department of Health. 


\title{
Conceptualising and treating social anxiety in autism spectrum disorder:
}

\section{A focus group study with multidisciplinary professionals}

\begin{abstract}
Background: Individuals who have autism spectrum disorders (ASD) commonly experience social anxiety (SA). Disentangling SA symptoms from core ASD characteristics is complex, partly due to diagnostic overshadowing and co-occurring alexithymia. Causal and maintaining mechanisms for SA in ASD are underexplored, but it is feasible that there is an ASD-specificity to the clinical presentation, with implications for the development of targeted treatments.

Method: We undertook five focus groups with multi-disciplinary professionals to investigate their perspectives about, and approaches to, working with individuals with ASD and SA. Data were analysed thematically.

Results: Data analysis revealed twooverarching themes: conceptualising SA in ASD; and service provision . Our results suggest that adaptations to service provision are pertinent, so as to accommodate inherent impairments which can mediate assessment and intervention.

Conclusions: Future studies should establish how aspects of the care pathway can be improved for individuals with ASD and SA.
\end{abstract}

\section{Keywords}

Autism spectrum disorder (ASD) - Asperger syndrome - social anxiety (SA) - social phobia - qualitative study 


\section{Introduction}

Autism spectrum disorders (ASD) are common lifelong neurodevelopmental conditions, characterised by socio-communication impairments, and engagement in circumscribed and routinised behaviour (WHO 1992). The ASD clinical presentation is markedly heterogeneous: symptoms can be subtle or severe, but they typically interfere significantly with daily functioning and result in long-term reliance on family members, and health and social care services (NICE 2012). A significant proportion of individuals with ASD have a concurrent intellectual disability (ID), which can markedly exacerbate functional impairment (NICE 2012). Mental health conditions often co-occur (Russell et al. 2015; Simonoff et al. 2008), affecting social and occupational functioning, and quality of life (Barneveld et al. 2014). Social anxiety (SA) in particular, is relatively common (Bejerot et al. 2014; Joshi et al. 2013; Kuusikko et al. 2008; Melfsen et al. 2006), with prevalence rates estimated as up to 50\% when assessed in child, adolescent and adult ASD samples (e.g. Bellini 2004; Maddox \& White 2015; Spain et al. in press).

Assessment, diagnosis, and treatment of SA in ASD, however, create several challenges for clinicians and researchers (Kreiser \& White 2014; Tyson \& Cruess 2012). First, both disorders are typified by difficulties with reciprocal interaction, reticence to initiate overtures, and avoidance of social situations (WHO 1992). This potentially makes delineating one condition from the other, and teasing apart the impact of either or both (e.g. on social functioning), a complex endeavour (Kerns \& Kendall 2012; White et al 2012). Second, methods commonly used to elicit information about mental health, such as self-report questionnaires, can prove difficult for individuals with ASD to understand, e.g. due to cooccurring alexithymia (i.e. difficulty reflecting on and reporting feelings; Bird \& Cook 2013); and self- and informant-ratings of SA in ASD are not necessarily concordant (Blakeley-Smith 
2012; Kuusikko et al. 2008). Further, the validity and reliability of SA (or proxy) measures in this clinical population are unknown (see Kreiser \& White 2014 for a comprehensive overview of the assessment of SA in ASD). This means that normative thresholds may not apply, and the likelihood of false negatives or positives may therefore be increased. Third, whether causal and maintaining mechanisms for SA in ASD are comparable to those reported for the typically developing (TD) population is yet to be established. It may be, for example, that ASD-specific factors, such as socio-communication deficits or difficulties tolerating change, increase vulnerability to or perpetuate these symptoms (e.g. Bellini 2006; Maddox \& White 2015; White et al. 2009). Finally, whether interventions such as cognitive behaviour therapy (CBT), the recommended treatment of choice for SA in TD samples (NICE 2013), is clinically useful for individuals with ASD (with or without a concurrent ID) has seldom been studied (Cardaciotto \& Herbert 2004; Wright 2013).

To date, studies investigating SA in ASD have predominantly used cross-sectional designs to examine prevalence rates or associations between SA and clinical or social outcomes, based on self- or informant-ratings. While a handful of previous studies have explored multidisciplinary team (MDT) professional experiences of working with individuals with ASD (e.g. Pellicano et al. 2014; Rogers et al. in press) no studies have focused specifically on SA. Little is known about approaches undertaken by clinicians to aid the process of assessment, conceptualisation of predisposing and perpetuating mechanisms, and treatment of these comorbid symptoms. Understanding the viewpoints of professionals may complement existing research about this topic, such as through contributing to the development of more honed, needs-led approaches to assessment and treatment. This study sought to ascertain professional perspectives about SA in ASD, and to establish how, if at all, clinicians and researchers adapt their practice when working with this clinical population. 


\section{Method}

\section{$\underline{\text { Study Design }}$}

A qualitative study design was employed. Focus groups, informed by a topic guide, were conducted.

\section{$\underline{\text { Participants }}$}

The study's sampling frame comprised MDT clinicians and researchers $(n=35)$ working in Greater London. We used a convenience sampling method, and contacted MDT professionals known to the research team. We specifically made a concerted attempt to approach professionals working with individuals with ASD (with or without ID) across the lifespan, and in a wide range of settings.Twenty-one individuals consented to take part; a $73.5 \%$ response rate. Reasons for non-participation were not systematically recorded. Clinicianparticipants included Adult Consultant Psychiatrists $(\mathrm{n}=5)$, Child and Adolescent Psychiatrists $(n=2)$, Speciality Doctors $(n=2)$, Clinical Psychologists $(n=2)$, Trainee Clinical Psychologists $(n=3)$, one CBT Therapist, and one Nurse Specialist. Clinicians worked across inpatient and outpatient settings. Six researchers participated, all of whom investigate biopsycho-social factors in neuro-developmental conditions. All participants had several years' experience of working with individuals with developmental disorders.

\section{$\underline{\text { Procedure }}$}

Five focus groups were conducted between January and March 2014. Focus groups took place in a quiet room, away from the clinical setting. Participants were able to choose when to attend from a range of dates and times. Each focus group involved between three and seven participants, and lasted for 52 to 100 minutes (mean duration $=79$ minutes). We 
conducted each focus group in the same way. Confidentiality issues (e.g. anonymising patient details and specific service locations) were discussed at the outset. A topic guide (available from the first author) was used as a basis for generating conversation. This included prespecified, semi-structured questions, such as "Do you think that people with ASD experience social anxiety? Is the clinical presentation different to social anxiety in non-ASD individuals? In what way do you think that social anxiety impacts on an individual's daily functioning? And what adaptations, if any, do you make to your standard clinical approach?". While a premise underpinning the focus groups was that individuals with ASD can experience SA, we asked questions which allowed for open-ended responses. Moreover, we sought to ensure that opinions about whether this may or may not be the case were equally vocalised. Prompts were also used as a means of encouraging discussion.

\section{Ethical Approvals}

NHS research ethics approvals were granted (REC ref. LO/13/0548). All participants provided written informed consent to take part, and for data to be disseminated.

\section{$\underline{\text { Data Analysis }}$}

Two researchers (LO'N and DS) listened to the audio-recordings, which were later transcribed verbatim by LO'N. We used a thematic analysis framework to analyse the data (Ritchie et al. 2014; Spencer et al. 2014). The analysis process comprised three stages: (1) initial themes were identified by 'indexing' the written transcripts, and these themes guided the formation of a framework within which transcribed materials were summarised; (2) key categories were listed to describe the data; and (3) patterns of association and difference were sought. To enhance methodological rigour, LO'N, FR, JS, and DS conducted the analysis independently. Analysis was performed until data saturation was reached, i.e. when no new 
themes or categories were identified Consensus was reached through discussion.

\section{Results}

Data analysis revealed that there were two overarching themes: conceptualising SA in ASD; and the care pathway. Please see Fig. 1 for a schematic representation of the themes and subthemes, and inter-dependent relationships between some of these.

\section{Theme 1: Conceptualising SA in ASD}

Focus group participants discussed two main aspects associated with the conceptualisation of SA in ASD: causal and maintaining mechanisms for SA symptoms; and development of a shared formulation with patients.

Sub-Theme 1.1: Causal and maintaining mechanisms for SA in ASD

Participants described a range of mechanisms that may be considered to cause and/or maintain SA symptoms. Interestingly, no clinicians (regardless of discipline) attributed the co-occurrence of ASD and SA solely to genetic or biological causes. Rather, SA was described as likely to be the result of ASD characteristics, psycho-social influences and systemic factors, with the weighting of these varying between individuals.

Sub-Theme 1.1.1: The influence of core ASD characteristics on SA development

There was unanimous consensus that ASD characteristics serve to increase vulnerability for anxiety and worries about social situations. These include the impact of socio-communication deficits on the ability to initiate and engage in reciprocal interaction, difficulties tolerating uncertainty in the face of unpredictable or unanticipated social situations, and sensory aversions that can render settings or physiological symptoms (in particular, anxiety or 
arousal) overwhelming.

I think that's what overwhelms them because to them every situation is completely new and different, because that's just the nature of social situations that you can't predict, and so I think it feels like an unsolvable problem for them, because they can't develop a rule that works every time. (FG3, F2)

Sometimes, just having a body that's just changing from some sort of arousal, can create a sense of aversion that I think is almost, that can almost trigger anxiety in a way ... (FG1, M1)

It was also hypothesised that individuals with particular subtypes of ASD may be more susceptible to developing SA, e.g. those individuals who are more socially motivated, and at the same time, more aware of their social difficulties. Further, SA may develop as a consequence of the increased demands and expectations placed upon individuals with ASD.

... because adults are more aware of their difficulties, so as a secondary factor, they start developing these symptoms of anxiety which eventually becomes a social anxiety disorder. (FG1, M1)

Sub-Theme 1.1.2: The impact of impairments or biases in cognitive processing Several participants remarked that for SA symptoms to develop, individuals must, to some extent, consider their own behaviour in the context of others, i.e. viewing themselves 'in the mind of others'. Regardless as to whether these considerations reflect reality or are biased (e.g. seeming more negative than neutral), this process relies on a degree of 'theory of mind' (ToM; the ability to recognise others' different thoughts and beliefs; Baron-Cohen et al. 
2001), which can be impaired in individuals with ASD.

... not being sure what the intentions are of other people ... they can't work out what their role is and what they're supposed to do, and other people seem to get it and they don't and it feels difficult. (FG3, M1)

Further, whereas 'the processing of self as a social object' (Clark 1999), maintains SA in TD individuals, this may not be the case in ASD.

... so it's that idea of performing a certain task, in front of others, and then being concerned about how they looked to other people. Which would imply to me, to some degree, a theory of mind, it's like imagining what that person, what that person's perspective might be of me right now, and I don't think that's the same as what would typically happen in autism. (FG3, M1)

Sub-Theme 1.1.3: The causal role of social adversity

Many individuals with ASD experience social stressors, such as bullying, rejection and ostracism. It was noted that these experiences occur across the lifespan. Understandably, this can incur anxiety about interactions.

... often they have lots of bad experiences with other people, they've been picked on or rejected, or bullied, or ostracised, or mocked. And often they struggle to understand what it is that they've done that has made them the butt of everybody else's jokes ... (FG5, M1)

Sub-Theme 1.1.4: The development of negative beliefs 
Negative beliefs about the self, e.g. relating to themes of inferiority and difference, may have a causal and maintaining role for SA. While socially anxious TD individuals often underestimate their abilities and are disproportionately concerned about their performance (Clark 1999), such beliefs may be congruent with the experiences of individuals with ASD.

... because most people I've seen have been anxious about social situations because they've messed up in the past, it's not like a thing that comes out of nowhere ... [They] start to internalise a belief that it's them that's at fault and that can make them anxious about performing. (FG2, F2)

Their built up experience with other people ... constantly not standing up to siblings, or to their parents expectations, or all of those things ... like the core belief of being not socially able is so strong in this group and across the board. (FG3, F2)

Sub-Theme 1.1.5: The influence of thinking styles on SA

Several participants stated that individuals with ASD can develop unhelpful ways of thinking, in particular, interpersonal sensitivity and paranoid thoughts; perhaps as a result of social adversity. Although this is unsurprising, this reinforces worries about others' intentions (however innocuous these may be) and encourages anticipatory anxiety.

... I certainly come across a lot of people who are really anxious around people because they don't know what they're thinking, so they start to assume people are against them, which kind of shifts into paranoia ... (FG2, F2)

It was also acknowledged that individuals with ASD are prone to perseveration and 
rumination. These thinking styles typically occur across situations. Although, on the one hand, there is utility in 'thinking things through', this can prove problematic, e.g. indirectly reinforcing doubt and a negative focus.

... it's more like a cycle, they feel anxious, they start thinking and ruminating about that and they become even more anxious and then it escalates to the moment they finally escape ... (FG1, M1)

Sub-Theme 1.1.6: Difficulties with emotion recognition and regulation

Some participants considered that difficulties with emotion recognition or regulation may be implicated in the development and maintenance of SA. For individuals with ASD, physiological arousal or anxiety may prove difficult to tolerate, e.g., because of a sensory aversion or sensitivity. Also, while individuals with ASD may notice that they 'feel physically different', they may lack the emotional literacy to explain why this is. Consequently, individuals may avoid situations that seem to make them feel uncomfortable, or they may choose to escape from anxiety-provoking situations.

I think that sometimes people can find it difficult just knowing what it is they're describing [emotions and feelings] ... but someone who doesn't know what they're feelings are could get really stuck ... (FG4, M1)

Sub-Theme 1.1.7: The impact of behavioural coping strategies

Overall, it was perceived that individuals likely develop multiple ways of coping with SA. While some coping strategies can prove helpful, other strategies such as avoidance of specific or general interactions and situations may unintentionally reinforce anxiety. It was noted 
however, that while avoidance is generally regarded to be an unhelpful 'safety behaviour', this may prove necessary for individuals with ASD because the need to avoid may be due to reasons other than anxiety (e.g. a sensory aversion).

But also when people are already in social situations that they have to manage, so working in a really noisy office where people are singing happy birthday all the time would be really difficult. So is it actually realistic for them to be able to go and sit somewhere else for half a day, rather than going 'no, you've got to get on with it, it's a safety behaviour. (FG2, F2)

Sub-Theme 1.1.8: Systemic Factors

The conceptualisation of SA in ASD requires an understanding of systemic factors, including the family context. For many individuals with ASD, family members provide ongoing care and support, well into adulthood. On occasion, there may be a functional basis for anxiety.

... it's not really necessarily an anxiety response, but some behaviours that are problematic are functional: it gets the family to come in and do something for them for example. So they [individuals with ASD] will have learned that there are certain things they don't know how to deal with and if they react in a certain way other people take care of it for them ... (FG3, M1)

Sub-Theme 1.2: Developing a shared formulation

A second sub-theme relating to the conceptualisation of SA in ASD concerned the development of a shared formulation, i.e. a road map for treatment. Acknowledgement of the multiple causal and maintaining mechanisms for SA has important implications for treatment (e.g. whether this should comprise pharmacological or psychological interventions, or both). Clinicians generally considered that a visual illustration of these mechanisms augments 
conversation. This is partly because individuals with ASD can experience difficulty with processing complex information, and also as they can be 'visual thinkers'. The complexity of the formulation, however, depends on an individual's emotional literacy.

I don't tend to find myself following a particular model because it's very rare that one will satisfactorily fit, so it's more idiosyncratic, and it will depend on the person's ability to tolerate lots of complex things, someone might be more visual, someone might like this, so don't [necessarily] draw a circle and an arrow. (FG3, M1)

\section{Theme 2: Service Provision}

Two sub-themes about service provision were identified: the care pathway; and clinician approaches to working with individuals with ASD.

\section{Sub-Theme 2.1: The Care Pathway}

Clinicians across focus groups perceived that access to services and the care pathway is far from straightforward, for several reasons: individuals with ASD or family members may not realise that social difficulties are attributable to a secondary cause; the parameters of UK National Health Service (NHS) commissioning structures appear rigid, primarily permitting the purchase of prescribed packages of care; and clinical and managerial gatekeepers may be uncertain about when to refer individuals on for mental health assessment, or they may be constrained by resources.

But what's difficult is when there's a service model that says work in this way, on this session you do this, by this session you've done that, and by that one you've finished your treatment, and you don't know whether the person's going to fit in that or not, and that can be true of 
anyone, but particularly in this group. (FG3, M1)

Sub-Theme 2.1.1: Assessment and diagnosis of SA

Clinicians considered that assessment and diagnosis of psychopathology in individuals with ASD can prove complex. This is particularly the case for SA (and indeed obsessive compulsive disorder; OCD) given that there is significant overlap in core and co-morbid signs and symptoms, i.e. diagnostic overshadowing. Hence, it is pragmatic to adopt a multifaceted approach to assessment.

... and often informant description is absolutely critical, you know the mother who knows the son very well, what's he like when he's anxious, becomes absolutely essential ... So I think what people say about themselves is important, and what informants say, I think observation is important, but also actually, critically behaviour, because a clear description of behaviour in social situations, it's often highly important. (FG5, M1)

Participants considered it important to 'look beyond' the initial presentation, as there may be discrepancies between symptoms described, and signs observed. Alexithymia, for example, can render it difficult for individuals with ASD to describe their emotions (or internal states). The implication is that assessment of SA should include information-gathering about beliefs and behaviours associated with SA, rather than just affect and physiological arousal.

I tend not to focus so much asking them how they feel because they usually don't respond very well, they don't describe it, so I prefer to be maybe a little more pedantic, so how I can describe one by one each symptom, but definitely I would ask them both for cognitive and physical symptoms. (FG1, M1) 
Methods of information-gathering in routine care typically comprise use of (screening) selfreport questionnaires and a clinical interview. The utility of self-report questionnaires for individuals with ASD was debated by participants, given that difficulties with introspection or perseveration may mean that this clinical population cannot easily complete a questionnaire. Thus, participants considered that assessment of SA in ASD should include attempts to ascertain specific examples about anxiety-provoking situations, with emphasis on antecedents and consequences.

... a diary could help, so you could ask someone for a week to write down every time they felt anxious - you might need to give them a better idea of what that meant [anxiety] ... (FG1, F1)

... be really concrete about giving an example, so 'when you were at this or when you were at the pub the other day what happened', and really trying to take them through it step by step. (FG3, F1)

Assessing the potential links between SA and sensory sensitivities was considered important, given that hypo- and hyper-sensitivities may be causally implicated.

... you do hear about people getting kind of sensory overload when they're in anxietyprovoking situations, so noise becoming more bothersome, and lights, and things like that ... it can be pretty distressing for people to be bombarded with all these things, and then that can make them act out, or act in a way that then other people are looking at them and then they are worried next time that it could happen again. (FG1, F1) 
Eliciting the extent to which symptoms impact on functioning or cause distress is paramount for diagnosis. This is particularly the case for the ASD population, because difficulties arising in the context of social situations may be attributable to either the core disorder, or comorbidity. Moreover, this has ramifications for treatment goals.

... whether it's causing them distress because if somebody is just not interested in talking to people that's one thing, but if they're upset about the fact they can't talk to people or talking to people really makes them feel anxious I suppose, if it makes them feel ways they don't like and that's causing them distress, then that seems more like anxiety than it does just autism (FG1, F1)

Sub-Theme 2.1.2: Goals and expectations for treatment It was perceived that individuals with ASD, family members, clinicians, and commissioners, have overlapping expectations about service provision but also, and crucially, these can sometimes differ. Individuals with ASD can want things to be, 'easier ... less anxietyprovoking', but this can prove difficult to conceptualise.

What a change looks like in their mind, it might be 'I have to be 100\% better and nothing's better until I've reached that point' but actually our whole job is pointing out the shades of grey ... (FG3, F1)

Conversely, not everyone wants things to change: the goal may be to maintain the 'status quo', which raises questions about 'best interests'. 
... part of what's at stake here is it's also a debate partly of what we think counts as 'a good life' or 'an acceptable life' that we should be encouraging people to have ... particularly in situations where you're saying to somebody who doesn't think that they've got a problem 'you have got a problem, that needs treatment'. (FG5, M1)

Participants described that the notion of change is best discussed with individuals with ASD, in a tentative and considered manner. As difficulties tolerating change and uncertainty are hallmark characteristics of ASD, it is pragmatic to introduce behavioural changes gradually; ideally, those that focus on the short- and medium-term.

So it's really getting the client to generate what actually functionally is getting in the way of them living their life and pick those things, rather than what would be in your ideal world. (FG3, F1)

Sub-Theme 2.1.3: Treatment for SA

No participants suggested that SA should solely be treated with medication. Rather, it was considered that treatment should include CBT. Several factors, however, potentially hinder engagement in, and the success of, psychological treatments. These include 'difficulties tolerating change and uncertainty', 'concrete thinking', 'concerns about what therapy entails', 'negative beliefs about performance', and 'difficulties perspective-taking ... ToM impairments'. Thus, interventions should be adapted in terms of structure and content.

You could have an adaptable therapy room, so you could send a pre-questionnaire "how do you like your lights, how do you like this" and it may be that they're all simple things that can be adjusted with the touch of a button. (FG3, M2) 
Pre-therapy interventions were deemed a necessary 'first step', including Motivational Interviewing techniques (Miller \& Rollnick 2013), psycho-education, and social skills interventions (SSI). These interventions are likely to serve as the basis from which individuals with ASD can use behavioural and cognitive techniques specifically targeting SA symptoms.

I think there's pre-therapeutic work to do just getting people to trust, and emotional recognition, labelling thoughts, understanding how it all links together. (FG2, F2)

...you do have to do a lot more sessions, a lot more of the initial work... you might have to take longer to do what you might call the assessment stage...you cannot go at the same pace that they've set it for [TD individuals]. (FG5, F1)

Therapists perceived that cognitive and behavioural interventions can ameliorate SA symptoms in this group, but there is no 'hard and fast rule' about which techniques work best.

So, in a way, probably what we're talking about is a kind of tool-kit of interventions that can be customised to a particular patient, where the skill of the therapist, actually, is critical. (FG4, M1)

Sub-Theme 2.1.4: Outcomes

Commissioning processes necessitate completion of generic outcome measures, yet these do not always reflect treatment gains given their broad remit, and they may have limited clinical utility for individuals with ASD. As such, there is a sense that individuals may have made 
gains, but these are difficult to quantify.

I was thinking outcomes are a very murky world because your outcome might be a couple of points on a anxiety scale, where it may be of no benefit to the person themselves, or the outcome might be how their life's changed, perhaps quality of life, so we get away from the feelings, the measurable change and the difference it's made. (FG3, M2)

Conversely, it was stated that sometimes individuals do not derive benefit from treatments offered, or it may be that as they 'do more', their anxiety increases.

I think the difficulty sometimes is you do a bit of work with somebody with autism and they start to learn more about how we feel, they recognise it even more. Or they start pushing themselves to do things that create more anxiety. And so they recognise it more. So they look like they're getting worse subjectively, but their world has got bigger. (FG2, F2)

\section{Sub-Theme 2.2: Clinician Approaches}

Focus group participants perceived that clinicians (and researchers) need to modify their clinical style and approach when working with individuals with ASD. Similar to clinical work with individuals with ID, it is important that clinicians are open-minded and flexible, i.e. being aware that there is a need to potentially adapt the structure, process and content of assessment or interventions, so as to make these more accessible and understandable. Modifications described included ensuring that appointments are offered at convenient times (e.g. a time that suits the patient), the clinical environment is not overly stimulating (e.g. not too brightly lit or noisy), conversation includes didactic questions as well as a socratic style, and that there is little reliance on metaphors or colloquialisms which may prove difficult for 
individuals with ASD to understand.

I'll ask them much more directly, and much more bluntly, and try and make sure my language isn't ambiguous and is very clear-cut ... (FG4, F1)

... it kind of feels a bit patronising if I say a metaphor or something and then say 'oh do you know what I mean by that?' and they go 'Yeah' - but I think it's worth checking because sometimes people say yeah but actually they don't know, and you've lost them ... (FG3, M1)

Additionally, it was noted that patients should be encouraged to be 'active participants', whereby their views about the pace and content of clinical work are sought. As such, individuals with ASD may have had limited opportunities to develop assertiveness skills. This means that an implicit element of the therapeutic relationship and process should involve encouraging patients to feel confident to say what they think.

... but sometimes emotions are not expressed so readily and easily, you can't read that somebody is happy, because their face looks exactly the same as when they are anxious ... I often ask for feedback, which doesn't always go down well, that's a bit like an unexpected and difficult question to answer, what do you want me to tell you ... but if I say 'was there anything we did today that you would like to do differently?', even still that can bring up questions, but I try and give them an opportunity to give feedback. (FG3, M1) 


\section{Discussion}

Using a qualitative study design, we undertook a series of focus groups with 21 MDT professionals and researchers, to explore opinions about SA in ASD. Data were analysed thematically, and transcripts were compared and contrasted. Two overarching themes emerged: conceptualising SA in ASD; and service provision.

Our study findings reflect that access to needs-led services is far from straightforward. The clinical implication is that needs for many individuals with ASD (who may also have an ID) remain unmet, symptoms become more chronic and entrenched, and the risk of additional problems increases; a common trajectory for TD individuals with SA (NICE 2013). Consequently, as mandated, commissioners and service providers should develop more equitable, accessible and flexible care pathways for individuals with ASD (DH 2010; HMO 2009; NICE 2011, 2012); the success of which is partly dependent on an appropriately skilled-up workforce. I

The perspectives of clinicians reported here highlight a concern that self-report questionnaires may lack sensitivity to detect SA symptoms in ASD (Brugha et al. 2015; Kreiser \& White 2014). Nevertheless, questionnaire completion may offer an insight into potential adjunctive difficulties, including alexithymia or perseveration, which may inform the clinical approach. Overall, our results indicate that assessment of SA in ASD should include information gleaned in multiple ways (NICE 2011, 2012; Tyson \& Cruess 2012).

Causal and maintaining mechanisms for SA in ASD were viewed as predominantly comprising psycho-social and systemic factors. Many of these - including negative social experiences, core beliefs relating to inferiority and difference, unhelpful ways of thinking and emotion dysregulation - are also evident in TD individuals with SA (Clark 1999; Morrison \& 
Heimberg 2013; Rapee \& Heimberg 1997). However, focus group participants hypothesised that several mechanisms may be unique to individuals with ASD. It is unsurprising that innate difficulties with social interaction, and verbal and non-verbal communication, serve as risk factors (White et al. 2009). Reflecting previous findings that an intolerance of uncertainty (IoU) is associated with anxiety in individuals with ASD (Boulter et al. 2014), our results suggest that the unpredictability and ambiguity of social situations may potentially activate IoU and thus, anxiety. Alternatively, SA may manifest as a learned response (Bellini 2006), whereby aspects of social situations are deemed (sensorily) aversive (e.g. noises or visual stimuli), culminating in avoidance. Over time, it may be that avoidance behaviour generalises across settings, partly because individuals with ASD cannot easily discriminate between situations. Further, it was proposed that SA may arise due to difficulties with recognising others' emotions and internal states; a finding which has been reported for TD samples (e.g. Morrison \& Heimberg 2013). In turn, this may heighten negative beliefs (e.g. about performance), or encourage a mistrust of others and paranoia (Spain et al. 2016). Bidirectionally, neuropsychological impairments and core ASD characteristics may indirectly increase the likelihood of peer rejection or ostracism (Schroeder et al. 2014; White \& Roberson-Nay 2009), and thus further entrench beliefs concerning inferiority. It may be that the risk of SA increases with age (e.g. more social demands during adolescence and adulthood), or when ASD is diagnosed later on in life (e.g. exacerbating a sense of difference and a situation of 'not knowing' the causes of socio-communication deficits). On the other hand, SA may be inversely related to the severity of core ASD symptoms (e.g. manifesting in those individuals who have more subtle deficits). Moreover, unlike CBT models of SA in TD samples, focus group participants perceived that self-focused attention and 'the processing of self as a social object' (Clark 1999) may not be a pivotal mechanism in ASD SA, perhaps due to inherent ToM (Baron-Cohen et al. 2001) or autobiographical memory deficits (Skirrow et 
al. 2014). Finally, our results also indicate that systemic factors may indirectly influence and reinforce SA symptoms. The social context and opportunities that individuals with ASD have are likely narrower than their TD counterparts (Howlin et al. 2013), and it may be that familial genetic, environmental or behavioural factors increase the risk for SA, as reported in TD samples (Rapee \& Heimberg 1997).

Our study cannot provide definitive conclusions about causal and maintaining mechanisms for SA in ASD. However, it emerged that clinicians see SA as stemming from a combination of neuropsychological, social, cognitive, behavioural, and affective processes, underpinned by the presence and impact of core ASD characteristics. The implication is that current SA models may require adaptation for this clinical population to incorporate the inherent impairments, present in individuals with ASD, which serve as risk factors. In practice, we suggest that treating clinicians use supervision to discuss case formulations, and to map out treatment plans. It may be pragmatic to emphasise the role of maintaining factors initially (so as not to overwhelm patients), and it may be that the process of formulation requires a slower pace. In addition, we suggest that when possible, systemic factors are evaluated as part of the assessment process, formulation of presenting problems (Tarrier \& Calam 2002), and treatment. With regard to treatment, our findings reinforce the need for 'pre-therapy' interventions (e.g. Gaus 2011; Spain et al. 2015). These may include emotional literacy sessions, and SSI to augment verbal and non-verbal communication (see also Anderson \& Morris 2006; Atwood 2004). Psychological treatment for SA in ASD probably requires behavioural, cognitive, and skills-based interventions; the weighting of techniques is best decided on a case-by-case basis. While this implies a move away from protocol-driven treatments, this does reflect the move toward employing transdiagnostic approaches (Mansell 
et al. 2009), and the need for modifications to standard CBT for this group, such as an extended course of treatment.

Focus group participants considered that clinicians should adapt their style when working with individuals with ASD, so as to accommodate possible impairments in expressive or receptive language, and cognitive capacity (Charman et al. 2011), and fear of negative evaluation characteristic of SA. Also, clinicians may need to focus intently (in the first instance), on developing trust, rapport and a sense of 'safety', perhaps in a similar way to when working therapeutically with individuals with psychosis (Holding et al. 2016). It is noteworthy that individuals with ASD may have had few adaptive peer relationships, and limited opportunities to develop confidence about social interactions, particularly with new people. This highlights the need for therapists to demonstrate a non-judgemental approach, but also more specifically, they should seek to provide and promote opportunities that explicitly denote empathy, validation and role modelling of appropriate social behaviour.

\section{$\underline{\text { Study Limitations }}$}

We acknowledge several study limitations. First, we recruited participants somewhat opportunistically. While several clinical disciplines were represented, it would have been interesting to hear also from other clinicians, e.g. speech and language therapists, or general health professionals working within primary care settings. Second, focus groups were facilitated by one person (DS), known to all participants, many of whom were also known to each other: existing working relationships may have indirectly affected the breadth or depth of participants' responses. Third, although smaller focus groups may generate less discussion, 
a comparison of the transcripts between our different focus groups revealed striking similarities in the codes and themes identified.

\section{$\underline{\text { Research Implications }}$}

We suggest that there is a need for quantitative and qualitative studies to investigate the phenomenology of SA in ASD. Whether SA differs according to ASD subtype (e.g. with versus without ID), or sex, warrants further investigation. Causal and maintaining mechanisms for SA in ASD are poorly understood; studies using cross-sectional and longitudinal designs would enhance our understanding of key mechanisms. Normative thresholds for commonly used SA measures should be established, and there is a clinical impetus for more reliable measures to be developed. Finally, intervention studies designed to target SA symptoms (in individuals who may also have an ID) are needed to determine which techniques (e.g. cognitive versus behavioural) are associated with improved outcomes.

\section{Conclusion}

Individuals with ASD are susceptible to developing anxiety about social situations and socialevaluative concerns. Assessment of SA in ASD requires a proactive approach, given that individuals may not necessarily help-seek, and their description of their difficulties may seem incongruent to their emotional expression. While there is preliminary evidence to support the use of CBT and psychological interventions to reduce SA symptoms in this clinical population, the study findings indicate that treatment requires a considered approach, an adapted clinical style, and more sessions than are typically commissioned. Further research is required to determine how aspects of the care pathway can be improved in order to accommodate the unique needs of individuals who have ASD and SA. 


\section{References}

Anderson S. \& Morris J. (2006) Cognitive behaviour therapy for people with Asperger syndrome. Behavioural and Cognitive Psychotherapy. Vol. 34 No. 3, pp. 293-303.

Attwood T. (2004) Cognitive behaviour therapy for children and adults with Asperger's syndrome. Behaviour Change, Vol. 21 No. 3, pp. 147-161.

Barneveld P. S. et al. (2014) Quality of life: A case-controlled long-term follow-up study, comparing young high-functioning adults with autism spectrum disorders with adults with other psychiatric disorders diagnosed in childhood. Comprehensive Psychiatry. Vol. 55 No.2, pp. 302-310.

Baron-Cohen S. et al. (2001) The "Reading the mind in the eyes" test revised version: a study with normal adults, and adults with Asperger syndrome or high-functioning autism. The Journal of Child Psychology and Psychiatry, Vol. 42 No. 2, pp. 241-251.

Bejerot S. et al. (2014) Social anxiety in adult autism spectrum disorder. Psychiatry Research. Vol. 220 No. 1, pp. 705-707.

Bellini S. (2004) Social Skill Deficits and Anxiety in High Functioning Adolescents with Autism Spectrum Disorders. Focus on Autism and Other Developmental Disabilities, Vol. 19 No. 17, pp. 78-86.

Bellini S. (2006) The Development of Social Anxiety in Adolescents with Autism Spectrum Disorders. Focus on Autism and Other Developmental Disabilities. Vol.21 No.3, pp. $138-145$

Bird G. \& Cook R. (2013) Mixed emotions: the contribution of alexithymia to the emotional symptoms of autism. Translational Psychiatry. Vol. 3 No. 7, pp. 1-8, e285.

Blakeley-Smith A. et al. (2012) Parent-child agreement of anxiety symptoms in youth with autism spectrum disorders. Research in Autism Spectrum Disorders. Vol. 6, pp. 707-716

Boulter C. et al. (2014) Intolerance of uncertainty as a framework for understanding anxiety in children and adolescents with autism spectrum disorders. Journal of Autism and Developmental Disorders. Vol. 44 No. 6, pp. 1391-402.

Brugha T. et al. (2015) Outcome measures in intervention trials for adults with autism spectrum disorders; a systematic review of assessments of core autism features and associated emotional and behavioural problems. International Journal of Methods in Psychiatric Research. Vol. 24 No. 2, pp, 99-115. 
Cardaciotto L. \& Herbert A. D. (2004) Cognitive behaviour therapy for social anxiety disorder in the context of Asperger's syndrome: A single-subject report. Cognitive and Behavioral Practice. Vol. 11 No. 1, pp. 75-81.

Charman T. et al. (2011) Defining the cognitive phenotype of autism. Brain Research. Vol. 1380, pp. 10-21.

Clark D. M. (1999) A Cognitive Perspective on Social Phobia. In: International Handbook of Social Anxiety: Concepts, Research and Interventions Relating to the Self and Shyness (Eds W. R. Crozier and L. E. Alden). pp. 405-430. John Wiley \& Sons Ltd., London.

Department of Health (2010) Implementing "Fulfilling and rewarding lives". Statutory guidance for local authorities and NHS organisations to support implementation of the autism strategy. pp. 1-29.

Gaus V. (2011) Cognitive behavioural therapy for adults with autism spectrum disorders. Advances in Mental Health and Intellectual Disabilities. Vol. 5 No. 5, pp. 15-26.

HM Government. (2009) Autism Act. UK.

Holding J. C. et al. (2016) Individuals' experiences and opinions of psychological therapies for psychosis: A narrative synthesis. Clinical Psychology Review. Vol. 43, pp.142161.

Howlin P. et al. (2013) Social outcomes in mid- to later adulthood among individuals diagnosed with autism and average nonverbal IQ as children. American Academy of Child and Adolescent Psychiatry. Vol. 52 No. 6, pp. 572-581.

Joshi G. et al. (2013) Psychiatric comorbidity and functioning in a clinically referred population of adults with autism spectrum disorders: a comparative study. Journal of Autism and Developmental Disorders. Vol. 43 No. 6, pp. 1314-1325.

Kerns C. M. \& Kendall P.C. (2012) The Presentation and Classification of Anxiety in Autism Spectrum Disorder. Clinical Psychology: Science and Practice. Vol. 19 No. 4, pp. 323-347

Kreiser N. \& White S.W. (2014) Assessment of Social Anxiety in Children and Adolescents with Autism Spectrum Disorder. Clinical Psychology Science and Practice. Vol. 21 No. 1, pp. 18-31.

Kuusikko S. et al. (2008) Social Anxiety in High-Functioning Children and Adolescents with Autism and Asperger Syndrome. Journal of Autism and Developmental Disorders. Vol. 38 No. 9, pp. 1697-1709. 
Maddox B. B. \& White S. W. (2015) Comorbid Social Anxiety Disorder in Adults with Autism Spectrum Disorder. Journal of Autism and Developmental Disorders. Vol. 45 No. 12 pp. 3949-3960.

Mansell W. et al. (2009) Conceptual Foundations of the Transdiagnostic Approach to CBT. Journal of Cognitive Psychotherapy. Vol. 23, No. 1, pp. 6-19.

Melfsen S. et al. (2006) The extent of social anxiety in combination with mental disorders. European Child and Adolescent Psychiatry. Vol. 15 No. 2, pp. 111-117.

Miller W. R. \& Rollnick S. (2013) Motivational Interviewing: Helping People Change. $3^{\text {rd }}$ Ed. The Guilford Press, New York.

Morrison A. S. \& Heimberg R. G. (2013) Social Anxiety and Social Anxiety Disorder. Annual Review of Clinical Psychology. Vol. 9, pp. 249-274.

National Institute for Health and Care Excellence (NICE) (2011) Autism in under 19s: recognition, referral and diagnosis. NICE guidelines [CG128].

National Institute for Health and Care Excellence (NICE) (2012) Autism: recognition, referral, diagnosis and management of adults on the autism spectrum. NICE guidelines [CG142].

National Institute for Health and Care Excellence (NICE) (2013) Social Anxiety Disorder: Recognition, Assessment and Treatment. NICE guidelines [CG159].

Pellicano E. et al. (2014) What should autism research focus upon? Community views and priorities from the United Kingdom. Autism, Vol. 18 No. 7, pp. 756-770.

Rapee R. M. \& Heimberg R.G. (1997) A cognitive-behavioral model of anxiety in social phobia. Behaviour Research and Therapy. Vol. 35 No.8, pp. 741-756.

Rogers C. L. et al. (2015, in press) Experiences of diagnosing autism spectrum disorder: a survey of professional in the United Kingdom. Autism: International Journal of Research and Practice (Sage). pp. 1-20.

Russell A. J. et al. (2015) The mental health of individuals referred for assessment of Autism Spectrum Disorder (ASD) in adulthood: A clinical report. Autism. pp. 1-5.

Russell E. \& Sofronoff K. (2005) Anxiety and social worries in children with Asperger syndrome. Australian and New Zealand Journal of Psychiatry. Vol. 39 No. 7, pp. 633-638.

Schroeder J. H. et al. (2014) Shedding Light on a Pervasive Problem: A Review of Research on Bullying Experiences among Children with Autism Spectrum Disorders. Journal of Autism and Developmental Disorders. Vol. 44 No. 7, pp. 1520-1534.

Simonoff E. et al. (2008) Psychiatric Disorders in Children with Autism Spectrum Disorders: Prevalence, Comorbidity, and Associated Factors in a Population-Derived Sample. 
Journal of American Academy of Child and Adolescent Psychiatry. Vol. 47 No. 8, pp. 921929.

Spain D. et al. (2015) Family therapy for autism spectrum disorders. Cochrane Database of Systematic Reviews. Issue. 10.

Spain D. et al. (2016) Conceptualising paranoia in ASD: A systematic review and development of a theoretical framework. Research in Autism Spectrum Disorders. Vol. 25, pp. 97-111.

Spain D. et al. Social anxiety in adult males with autism spectrum disorders. Research in Autism Spectrum Disorders. (in press)

Tarrier N. \& Calam R. (2002) New developments in cognitive-behavioural case formulation. Epidemiological, systemic and social context: An integrative approach. Behavioural and Cognitive Psychotherapy. Vol. 30 No. 3, pp. 311-328.

Tyson K. E. \& Cruess D. G. (2012) Differentiating high-functioning autism and social phobia. Journal of Autism and Developmental Disorders. Vol. 42 No. 7, pp. 1477-1490.

White S. W. et al. (2009) Anxiety in children and adolescents with autism spectrum disorders. Clinical Psychology Review. Vol. 29 No. 3, pp. 216-229.

White S. W. \& Roberson-Nay R. (2009) Anxiety, Social Deficits, and Loneliness in Youth with Autism Spectrum Disorders. Journal of Autism and Developmental Disorders, Vol. 39, pp.1006-1013.

White S.W. et al. (2012) Examining Shared and Unique Aspects of Social Anxiety Disorder and Autism Spectrum Disorder Using Factor Analysis. Journal of Autism Developmental Disorders. Vol.42 No. 5, pp. 874-884.

WHO (1992) ICD-10. WHO.

Wright K. (2013) Cognitive behavioural therapy for anxiety in a man with autism spectrum disorder, intellectual disability, and social phobia. Advances in Mental Health and Intellectual Disabilities, Vol. 7 No. 5, pp. 284-292. 
Figure 1: Thematic framework

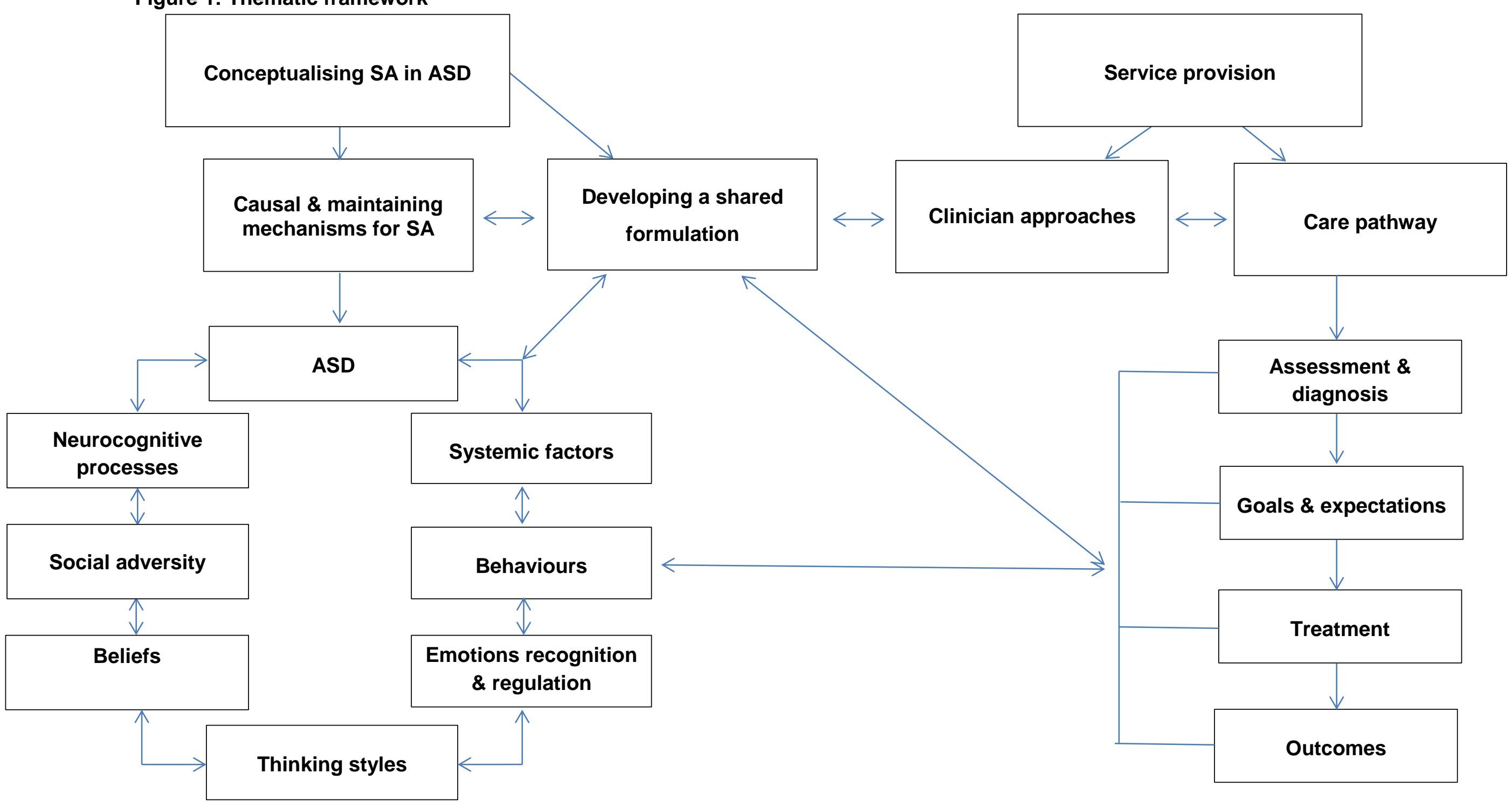

